\title{
THE FREQUENCY OF CARBON STARS AMONG EXTREMELY METAL-POOR STARS ${ }^{1}$
}

\author{
Judith G. Cohen, ${ }^{2}$ Stephen Shectman ${ }^{3}$ Ian Thompson, ${ }^{3}$ Andrew McWilliam, ${ }^{3}$ Norbert Christlieb, \\ Jorge Melendez, ${ }^{2}$ Franz-Josef Zickgraf, ${ }^{4}$ Solange Ramírez, ${ }^{5}$ And Amber Swenson ${ }^{2}$ \\ Received 2005 March 29; accepted 2005 October 4; published 2005 October 21
}

\begin{abstract}
We demonstrate that there are systematic scale errors in the $[\mathrm{Fe} / \mathrm{H}]$ values determined by the Hamburg/ESO Survey (HES; and by inference by the HK survey in the past) for certain extremely metal-poor, highly C-enhanced giants. The consequences of these scale errors are that (1) the fraction of carbon stars at extremely low metallicities has been overestimated in several papers in the recent literature, (2) the number of extremely metal-poor stars known is somewhat lower than has been quoted in the recent literature, and (3) the yield for extremely metalpoor stars by the HES is somewhat lower than is stated in the recent literature. A preliminary estimate for the frequency of carbon stars among the giants in the HES sample with $-4<[\mathrm{Fe} / \mathrm{H}]<-2.0$ dex is $7.4 \% \pm 2.9 \%$; adding an estimate for the $\mathrm{C}$-enhanced giants with $[\mathrm{C} / \mathrm{Fe}]>1.0$ dex without detectable $\mathrm{C}_{2}$ bands raises the fraction to $14 \% \pm 4 \%$. We rely on the results of an extensive set of homogeneous, detailed abundance analyses of stars expected to have $[\mathrm{Fe} / \mathrm{H}] \leq-3.0$ dex selected from the HES to establish these claims. We have found that the Fe metallicity of the cooler $\left(T_{\text {eff }} \lesssim 5200 \mathrm{~K}\right) \mathrm{C}$ stars as derived from spectra taken with HIRES at Keck are a factor of $\sim 10$ higher than those obtained via the algorithm used by the HES project to analyze the moderateresolution follow-up spectra, and this algorithm is identical to that used until very recently by the HK survey. This error in the Fe abundance estimate for $\mathrm{C}$ stars arises from a lowering of the emitted flux in the continuum bandpasses of the KP index (Ca II $\lambda 3933$ ) and, particularly, the HP2 (H $\delta$ ) index used to estimate [Fe/H] due to absorption from strong molecular bands.
\end{abstract}

Subject headings: Galaxy: abundances — Galaxy: halo — stars: carbon

Online material: color figure

\section{INTRODUCTION}

We are engaged in a large-scale project to find additional extremely metal-poor stars in the halo of our Galaxy. The major existing survey for very metal-poor stars is the HK survey, described in detail by Beers et al. (1985, 1992). The stellar inventory of this survey has been scrutinized with considerable care over the past decade, but, as summarized by Beers et al. (1998), only roughly 100 are believed to be extremely metalpoor (EMP), with $[\mathrm{Fe} / \mathrm{H}] \leq-3.0$ dex. ${ }^{6}$ We are therefore exploiting the database of the Hamburg/ESO Survey (HES) for this purpose. The HES is an objective prism survey from which it is possible to efficiently select EMP stars (Christlieb 2003). The existence of a new list of candidates for EMP stars with $[\mathrm{Fe} / \mathrm{H}]<-3$ dex selected in an automated and unbiased manner from the HES, coupled with the very large collection area and efficient high-resolution echelle spectrographs of the new generation of large telescopes, offers us the possibility for a large increase in the number of EMP stars known and for a better understanding of their properties.

\footnotetext{
${ }^{1}$ Based in part on observations obtained at the W. M. Keck Observatory, which is operated jointly by the California Institute of Technology, the University of California, and the National Aeronautics and Space Administration.

${ }^{2}$ Palomar Observatory, Mail Stop 105-24, California Institute of Technology, Pasadena, CA 91125; jlc@astro.caltech.edu, jorge@astro.caltech.edu, aswenson@its.caltech.edu

${ }^{3}$ Carnegie Observatories, 813 Santa Barbara Street, Pasadena, CA 91101; shec@ociw.edu, ian@ociw.edu, andy@ociw.edu.

${ }^{4}$ Hamburger Sternwarte, Universität Hamburg, Gojenbergsweg 112, D-21029, Hamburg, Germany; nchristlieb@hs.uni-hamburg.de, fzickgraf@hs.uni-hamburg.de.

${ }^{5}$ Spitzer Science Center, Mail Stop 100-22, California Institute of Technology, Pasadena, CA 91125; solange@ipac.caltech.edu.

${ }^{6}$ The standard nomenclature is adopted; the abundance of element $\mathrm{X}$ is given by $\epsilon(\mathrm{X})=N(\mathrm{X}) / N(\mathrm{H})$ on a scale in which $N(\mathrm{H})=10^{12} \mathrm{H}$ atoms. Then $[\mathrm{X} / \mathrm{H}]=\log _{10}[N(\mathrm{X}) / N(\mathrm{H})]-\log _{10}[N(\mathrm{X}) / N(\mathrm{H})]_{\odot}$, and similarly for $[\mathrm{X} / \mathrm{Fe}]$.
}

With the High Resolution Echelle Spectrometer (HIRES; Vogt et al. 1994) at the Keck I Telescope, we have obtained and analyzed spectra of a large number of EMP candidates selected from the HES. The normal procedures outlined by Christlieb (2003) to isolate EMP stars from the candidate lists produced by the HES were followed. In an effort to avoid selection biases, these differ from the criteria adopted by the HK survey (see Christlieb 2003 for details). Candidate EMP stars selected from the HES were vetted via moderate-resolution spectroscopy at large telescopes to eliminate the numerous higher abundance interlopers. Most of the follow-up spectra for the stars discussed here were obtained with the Double Spectrograph (DBSP; Oke \& Gunn 1982) on the 200 inch Hale Telescope at Palomar Mountain, denoted P200 (a few are from the Boller \& Chivens spectrograph on the Clay and Baade Telescopes at the Las Campanas Observatory) during the period from 2001 to the present. We intend to observe all candidates to the magnitude limit of the HES $(B \sim 17.5)$ in our fields; observations are now complete in $\sim 990 \mathrm{deg}^{2}$, complete to $B=16.5$ in an additional $\sim 700 \mathrm{deg}^{2}$, and approaching completion in the remaining fields.

These follow-up spectra are used to determine an accurate measure of the metallicity of the star, much more so than is possible to derive from the low-resolution objective prism spectra of the HES itself. This is accomplished via a combination of the strength of absorption in $\mathrm{H} \delta$ (the HP2 index, which determines $T_{\text {eff }}$ ) and in the Ca II line at $3933 \AA$ (the KP index, which determines $[\mathrm{Fe} / \mathrm{H}]$, once $T_{\text {eff }}$ and hence $\log g$ are specified). A calibration between the strength of the indices and metallicity is required, and is generally derived from literature searches for high-resolution abundance studies of relevant stars. We denote the resulting metallicity value as $[\mathrm{Fe} / \mathrm{H}](\mathrm{HES})$. The specific algorithm adopted by the HES is described in Beers et al. (1999) and is essentially identical to that used by the HK 


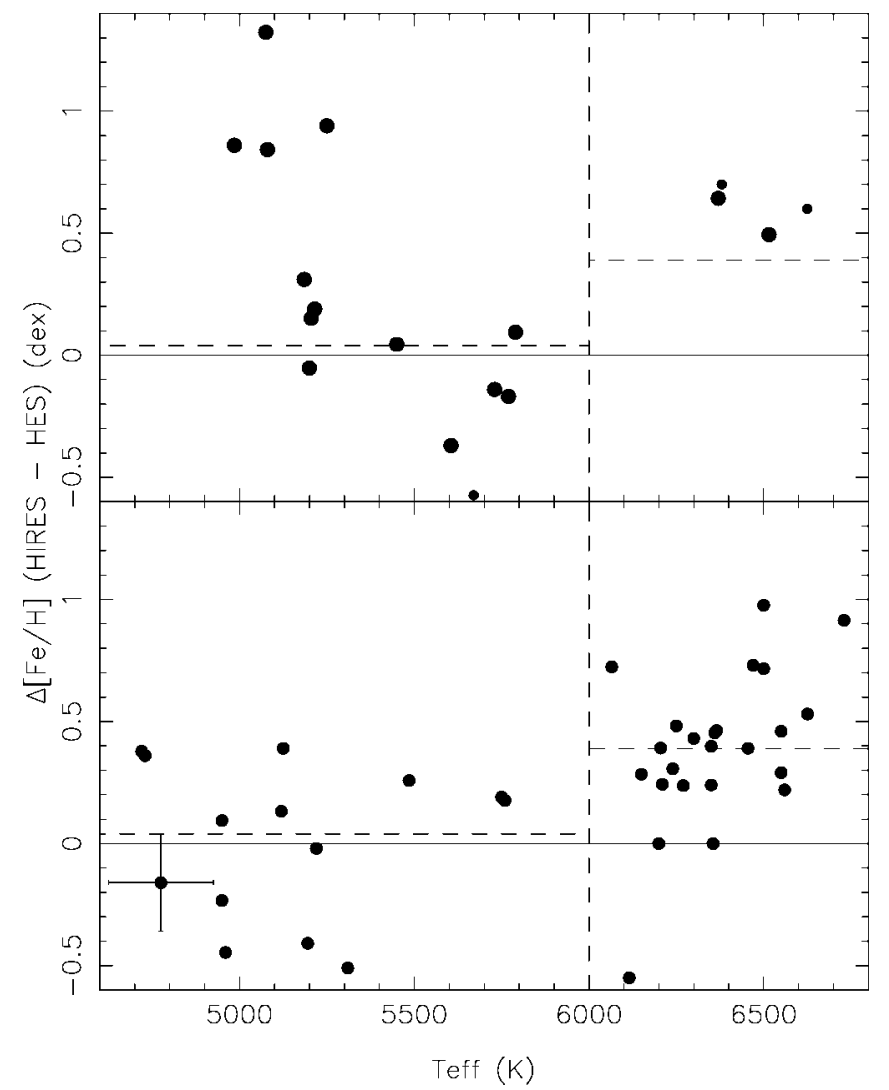

FIG. 1.-The difference between [Fe/H](HES) and [Fe/H](HIRES) is shown as a function of $T_{\text {eff }}$ for the C stars (upper panel; the smaller symbols denote C-enhanced stars) and for the C-normal stars (lower panel) for those EMP candidates from the HES with analyses based on Keck/HIRES spectra. The vertical dashed line separates the giants from the dwarfs, while the horizontal dashed lines represent the mean $\Delta$ for the C-normal giants and for the Cnormal dwarfs. A typical error for a C-normal giant is shown.

survey until recently; the latest updates to the algorithm as used by the HK survey are described in Rossi et al. (2005).

\section{SYSTEMATIC CALIBRATION PROBLEMS IN THE METALLICITY SCALE OF THE HES AND THE HK SURVEY}

Stars were chosen for observation at high resolution with HIRES primarily on the basis of low predicted metallicity; every star with $[\mathrm{Fe} / \mathrm{H}](\mathrm{HES}) \leq-2.9$ dex north of $\delta-25^{\circ}$ was put on the HIRES observing list. Spectra have now been obtained for more than 55 EMP candidates from the HES. In all cases, the stellar parameters have been determined by J. G. C. from broadband $(V-I, V-J$, and $V-K)$ photometry and theoretical isochrones with no reference to the spectra themselves. Insofar as possible, the procedures, the codes, the model atmospheres (we use those of Kurucz 1993), and the atomic data used to reduce the HIRES echelle spectra and to carry out the detailed abundance analysis are identical, and the analyses are thus as homogeneous as possible. Cohen et al. (2004) present full details of the analysis and results for a large sample of EMP dwarfs from the HES, while J. G. Cohen et al. (2005, in preparation) will present abundance analyses for 15 of the 16 known carbon stars from this HES sample; one of these has not yet been observed with HIRES. Fifteen C-normal giants have been analyzed to date, and these will appear in a future publication (J. G. Cohen et al. 2006a, in preparation).

Our operational definition of a carbon star (C star) is one whose spectrum shows bands of $\mathrm{C}_{2}$. The P200 DBSP spectra mostly extend to $5300 \AA$; hence, the prominent $\mathrm{C}_{2}$ band at

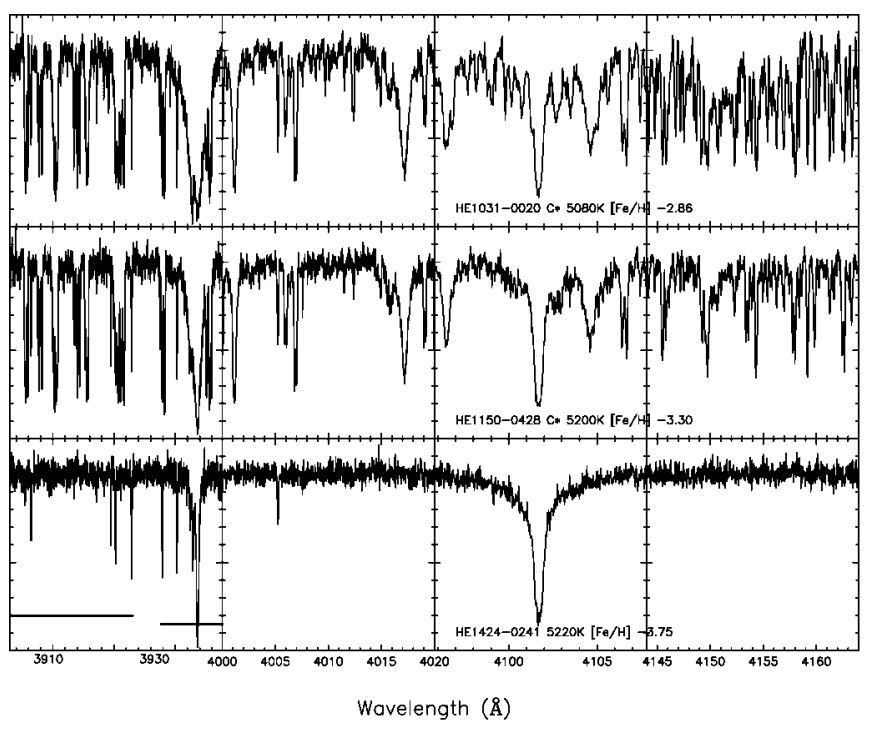

FIG. 2.-Four spectral regions from the HIRES spectra of two C stars (two upper rows) and an C-normal EMP giant. Each star has $T_{\text {eff }} \sim 5150 \mathrm{~K}$, and each has $[\mathrm{Fe} / \mathrm{H}](\mathrm{HES})<-3.2$ dex. The HIRES-derived $[\mathrm{Fe} / \mathrm{H}]$ are shown in the label for each star. The leftmost column shows the region of the blue continuum and the feature bandpass for the KP index as defined in Beers et al. (1999); these are indicated by the horizontal lines in the bottom left panel. The next column is the red continuum bandpass for the KP index, which is also the blue continuum bandpass for the HP2 index. The column after that shows the feature bandpass for the HP2 index measuring the strength of $\mathrm{H} \delta$. The rightmost column is the red continuum bandpass for the HP2 index, which is heavily contaminated by molecular features in the $\mathrm{C}$ stars. The feature bandpasses are taken as $12 \AA$ wide. The vertical scale is identical for each panel, $0.0-1.2$ for the normalized flux.

$5160 \AA$ is included. We are reasonably certain, as will be discussed in J. G. Cohen et al. (2006b, in preparation), through inspection of the regions of both $\mathrm{C}_{2}$ and $\mathrm{CH}$ in these followup spectra, that there are no additional giant $\mathrm{C}$ stars in the Palomar sample. If no $\mathrm{C}_{2}$ bands are detected, but $[\mathrm{C} / \mathrm{Fe}]>$ 1 dex, we denote a star to be C-enhanced. Also, we denote stars with $T_{\text {eff }}>6000 \mathrm{~K}$ as "dwarfs," while all cooler stars are called "giants."

The difference between the $[\mathrm{Fe} / \mathrm{H}]$ derived from analysis of the HIRES spectrum versus that obtained by applying the algorithm of Beers et al. (1999) to the moderate-resolution follow-up spectra for the set of 497 candidate EMP stars with moderate-resolution follow-up P200 spectra is shown in Figure 1. The giants with normal $\mathrm{C}$ and the warmer $\mathrm{C}$ giants show good agreement; $[\mathrm{Fe} / \mathrm{H}](\mathrm{HES})$ inferred from the moderateresolution spectra is a reliable indicator of the Fe metallicity found from analysis of HIRES spectra. Getting the dwarfs correct is harder as metal lines become weaker at their higher $T_{\text {eff }}$. Also, we have adopted a $T_{\text {eff }}$ scale for them that is hotter than that used in most earlier analyses that provided the calibration of the Beers et al. (1999) relation for EMP dwarfs (see, e.g., Norris et al. 1996 and Ryan et al. 1996). In the mean, $[\mathrm{Fe} / \mathrm{H}](\mathrm{HES})$ systematically underestimates our HIRES-based Fe metallicity by 0.37 dex for EMP dwarfs, corresponding to a systematic difference in adopted $T_{\text {eff }}$ of $\sim 400 \mathrm{~K}$.

However, Figure 1 shows that for the cooler $\mathrm{C}$ giants $\left(T_{\text {eff }} \lesssim\right.$ $5200 \mathrm{~K}),[\mathrm{Fe} / \mathrm{H}](\mathrm{HES})$ substantially underestimates our HIRESbased Fe metallicity by $\sim 1$ dex. Preston \& Sneden (2001) suggested the presence of a systematic error of comparable size (up to $1 \mathrm{dex}$ ) for the sample of $\mathrm{C}$ stars they analyzed from the $\mathrm{HK}$ survey. This is a very large systematic error, much too large to be caused by problems in the $T_{\text {eff }}$ scale, and so we attempt to understand what might be causing it. In Figure 2, we show sections 
of the HIRES spectra of three EMP candidates from the HES shifted into the rest frame. These stars all have $T_{\text {eff }} \sim 5150 \mathrm{~K}$. Two are $\mathrm{C}$ stars, and the third is a genuine EMP giant with weak $\mathrm{CH}$. Each of these stars has $[\mathrm{Fe} / \mathrm{H}](\mathrm{HES})<-3.2$ dex. The red and blue continuum and the feature bandpass are shown for the $\mathrm{KP}$ and HP2 indices used to determine $[\mathrm{Fe} / \mathrm{H}](\mathrm{HES})$, with a feature bandpass $12 \AA$ wide for each (see Beers et al. 1999 for details of the index definitions). The figure clearly shows the source of the problem afflicting the C stars-the "continuum" bands are full of strong molecular absorption, particularly the red continuum band for the HP2 index. If the HP2 index is underestimated because the continuum is depressed, then the star is assumed to be cooler than it actually is, and the resulting $[\mathrm{Fe} / \mathrm{H}](\mathrm{HES})$ for a fixed KP index will be too low. Furthermore, the blue continuum region of the $\mathrm{KP}$ index also shows strong $\mathrm{CH}$ absorption (the big chunks missing from the spectra of the $\mathrm{C}$ stars in the left column blueward of $\mathrm{Ca}$ II $\lambda$ 3933), and hence the abundance indicator KP will also be underestimated. The derived $[\mathrm{Fe} / \mathrm{H}](\mathrm{HES})$ obtained using the calibration of Beers et al. (1999) will thus be substantially reduced below its true value for such $\mathrm{C}$ stars. Because the absorption in the relevant spectral regions arises from both $\mathrm{CN}$ and $\mathrm{CH}$, the magnitude of this effect depends on additional factors such as the $\mathrm{C}$ enhancement and the $\mathrm{C} / \mathrm{N}$ ratio, as well as on $T_{\text {eff }}$.

Several tests have been performed to verify this. First we checked that the measured KP and HP2 indices for $\mathrm{C}$ stars (and for C-normal stars) can be reproduced to within their uncertainties from the much more precise HIRES spectra. We also checked that adding back the missing flux removed from the continuum by absorption in the sidebands significantly increases the KP index and, particularly, the HP2 index above the measured values, by factors of 2 or more. Finally, we checked that by so altering the KP and HP2 indices, we derive a significantly higher value for $[\mathrm{Fe} / \mathrm{H}](\mathrm{HES})$ that is much closer to that obtained by the detailed abundance analyses for the cooler C stars.

\section{DISCUSSION AND IMPLICATIONS}

An underestimate of a factor of $\sim 1$ dex in the deduced value of $[\mathrm{Fe} / \mathrm{H}](\mathrm{HES})$ for the cooler $\mathrm{C}$ stars will have significant effects. Figure 3 shows [Fe/H](HES) versus $V-K$ for a sample of 489 EMP candidates from the HES with moderate-resolution spectra from the DBSP at the Hale Telescope (details will appear in J. G. Cohen et al. 2006b, in preparation). The 10 known $\mathrm{C}$ stars and the one C-enhanced star with HIRES analyses ${ }^{7}$ from this sample are indicated. In the upper panel these stars are plotted at their $[\mathrm{Fe} / \mathrm{H}](\mathrm{HES})$ values, while in the lower panel they are plotted at their $[\mathrm{Fe} / \mathrm{H}](\mathrm{HIRES})$ as determined from detailed abundance analyses. ${ }^{8}$ Although at their nominal $\mathrm{Fe}$ metallicities the $\mathrm{C}$ stars dominate the population of the giants below $[\mathrm{Fe} / \mathrm{H}](\mathrm{HES})-3$ dex, using the results from analysis of high-resolution spectra in the lower panel, the frequency of $\mathrm{C}$ stars among the most EMP stars is considerably reduced.

It is our contention that, as shown in Figure 3, this underestimate of the metallicity of the cooler $\mathrm{C}$ stars by the algorithm of Beers et al. (1999), used by both the HES and in the past by the HK survey, produces a spurious high frequency of $\mathrm{C}$ stars among EMP stars (see Fig. 1). Using the [Fe/H](HES) values for the $\mathrm{C}$ stars would yield an apparent $\mathrm{C}$ star fraction of $33 \%$ for $[\mathrm{Fe} / \mathrm{H}] \leq-3$ dex, while using the HIRES Fe metallicities, a value a factor of 2.4 smaller is obtained. There are 122 HES

\footnotetext{
${ }^{7}$ The C-enhanced star is HE 0024-2523 (see Lucatello et al. 2003 and references therein).

${ }^{8}$ Two of the $\mathrm{C}$ stars in the P200 sample have not been observed with HIRES; they are shown with the appropriate offset determined from Fig. 1.
}

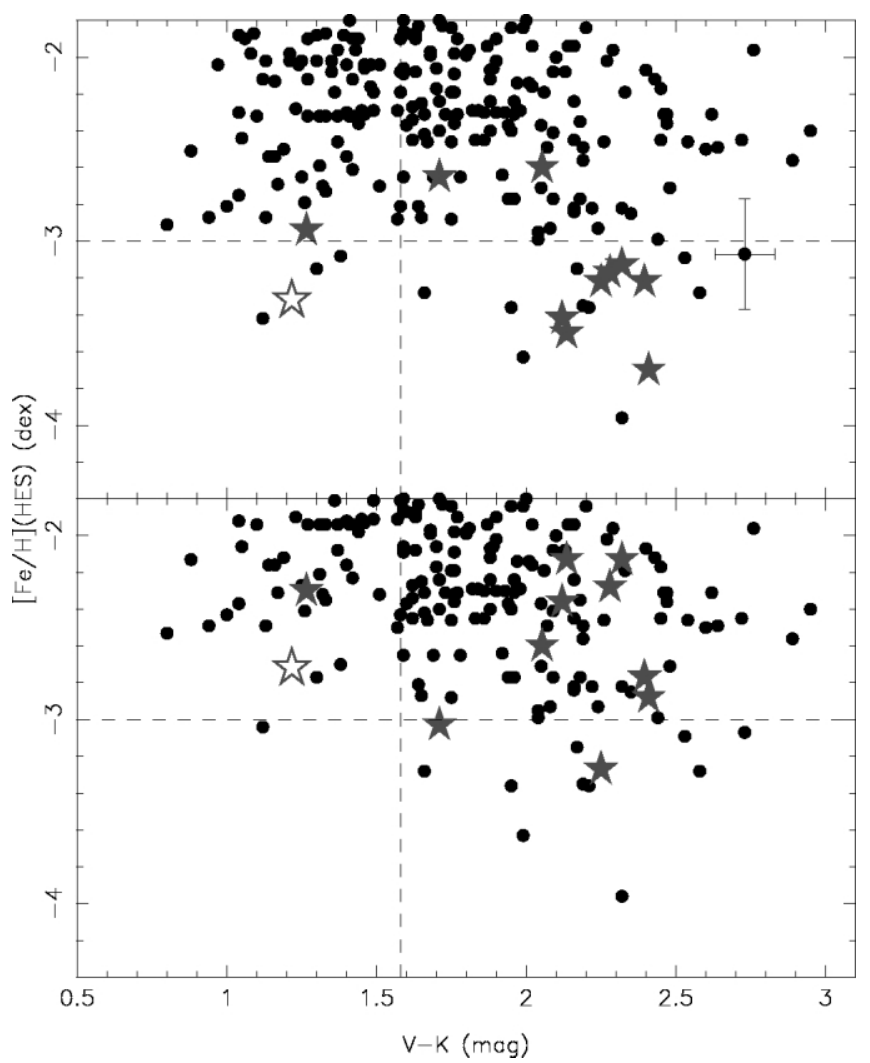

FIG. 3.-Plot of [Fe/H](HES) vs. $V-K$ for a sample of 489 EMP candidates from the HES with moderate-resolution spectra from the DBSP at the Hale Telescope (filled circles, limited to stars with $[\mathrm{Fe} / \mathrm{H}](\mathrm{HES})<-1.8 \mathrm{dex}$ ). The $\mathrm{C}$ stars from this sample are indicated by filled stars; the C-enhanced star is shown as an open star. In the upper panel the $\mathrm{C}$ stars are plotted at their $[\mathrm{Fe} / \mathrm{H}](\mathrm{HES})$ values, while in the lower panel they are plotted at their $[\mathrm{Fe} / \mathrm{H}](\mathrm{HIRES})$ values. The C-normal dwarfs have also been shifted toward higher $[\mathrm{Fe} / \mathrm{H}]$ by 0.38 dex in the lower panel. A typical error for a EMP giant with normal $\mathrm{C}$ is shown for a single star in the upper panel. The vertical dashed line separates the giants from the dwarfs. The horizontal dashed line indicates the EMP cutoff at $[\mathrm{Fe} / \mathrm{H}]=-3.0 \mathrm{dex}$. [See the electronic edition of the Journal for a color version of this figure.]

giants with $[\mathrm{Fe} / \mathrm{H}](\mathrm{HES})<-2.0$ dex in our sample, suggesting a C star frequency of $7.4 \% \pm 2.9 \%$ for EMP stars. Adding in the fraction of C-enhanced stars among giants with $[\mathrm{Fe} / \mathrm{H}](\mathrm{HES})$ $<-2.0$ dex found by Cohen et al. (2005) of $6.5 \% \pm 2.7 \%$, one obtains a total fraction of C-rich stars with $[\mathrm{C} / \mathrm{Fe}]>1.0$ dex of $14 \% \pm 4 \%$ among the giants in our HES EMP sample with $[\mathrm{Fe} / \mathrm{H}](\mathrm{HES})<-2.0$ dex, smaller than the value of $25 \%$ for stars with $[\mathrm{Fe} / \mathrm{H}]<-2.5$ dex given by Marsteller et al. (2005). (We have derived this fraction for the C-enhanced stars among the dwarfs in our sample with $[\mathrm{Fe} / \mathrm{H}](\mathrm{HES})<-2.0 \mathrm{dex}$; it has the same value, as will be reported in J. G. Cohen et al. 2006b, in preparation.) We are currently analyzing larger samples to refine this fraction. It will probably be necessary to include an indicator of the strength of the molecular bands (most easily the $\mathrm{G}$ band of $\mathrm{CH}$, i.e., the GP index already introduced in Beers et al. 1985) with the standard KP and HP2 indices in the calibration algorithm to obtain valid $\mathrm{Fe}$ abundances and maximum information from the HES and HK survey samples and/or to replace the HP2 index with a combination of $V$ from the HES and $J$ or $K$ from the Two Micron All Sky Survey; the latter was not available at the time the HK survey defined their metallicity calibration algorithms (see, e.g., Rossi et al. 2005).

The metallicity distribution function is very sharply declining among halo stars at the lowest Fe metallicities. Thus, the systematic errors we have found in the calibration of the HES, 
and by inference the HK metallicity scale of Beers et al. (1999), at least until quite recently (see Rossi et al. 2005), will also lead directly to systematic overestimates of the number of EMP stars and of the yield for EMP stars by these two major surveys. We are currently evaluating in detail the impact of these calibration errors on such issues.

The entire Keck/HIRES user community owes a huge debt to the many other people who have worked to make the Keck
Telescope and HIRES a reality and who operate and maintain the Keck Observatory. J. G. C. and J. M. are grateful for partial support from NSF grant AST-0205951. J. G. C. is grateful for support from the Ernest Fullam Award of the Dudley Observatory that helped initiate this work. The work of N. C. and F.-J. Z. is supported by Deutsche Forschungsgemeinschaft (grants Ch 214-3 and Re 353/44). N. C. acknowledges support through a Henri Chretien International Research Grant administered by the American Astronomical Society.

\section{REFERENCES}

Beers, T. C., Preston, G. W., \& Shectman, S. 1985, AJ, 90, 2089 1992, AJ, 103, 1987

Beers, T. C., Rossi, S., Norris, J. E., Ryan, S., Molaro, P., \& Rebolo, R., 1998, Space Sci. Rev., 84, 139

Beers, T. C., Rossi, S., Norris, J. E., Ryan, S., \& Shefler, T. 1999, AJ, 117, 981

Christlieb, N. 2003, Rev. Mod. Astron., 16, 191

Cohen, J. G., et al. 2004, ApJ, 612, 1107

2005, in IAU Symp. 228, From Lithium to Uranium: Elemental Tracers of Early Cosmic Evolution, ed. V. Hill, P. Francois, \& F. Primas (Cambridge: Cambridge Univ. Press), in press

Kurucz, R. L. 1993, CD-ROM 13, ATLAS9 Stellar Atmosphere Programs and $2 \mathrm{~km} / \mathrm{s}$ Grid (Cambridge: SAO)
Lucatello, S., Gratton, R., Cohen, J. G., Beers, T. C., Christlieb, N., Carretta, E., \& Ramírez, S. 2003, AJ, 125, 875

Marsteller, B., Beers, T. C., Rossi, S., Christlieb, N., Bessell, M., \& Rhee, J., 2005, Nucl. Phys. A, in press

Norris, J. E., Ryan, S. G., \& Beers, T. C. 1996, ApJS, 107, 391

Oke, J. B., \& Gunn, J. E. 1982, PASP, 94, 586

Preston, G. W., \& Sneden, C. 2001, AJ, 122, 1545

Rossi, S., Beers, T. C., Sneden, C., Sevastyanenko, T., Rhee, J., \& Marsteller, B. 2005, AJ, in press (astro-ph/0508202)

Ryan, S. G., Norris, J. E., \& Beers, T. C. 1996, ApJ, 471, 254

Vogt, S. E., et al. 1994, Proc. SPIE, 2198, 362 